Journal of Mathematics and Informatics

Vol. 8, 2017, 19-24

ISSN: 2349-0632 (P), 2349-0640 (online)

Published 11 July 2017

www.researchmathsci.org

DOI: http://dx.doi.org/10.22457/jmi.v8a3

Journal of

Mathematics and

Informatics

\title{
Research on Oscillation Properties for a Class of the Second-order Mixed Partial Difference Equations
}

\author{
Hui-li Ma ${ }^{1}$ and Hai-xia Wang ${ }^{2}$
}

${ }^{1}$ College of Business, Northweat Normal University, Lanzhou-730070, Lanzhou, China.

E-mail: mahuili@nwnu.edu.cn

${ }^{2}$ College of Mathematics and Statistics, Northweat Normal University, Lanzhou-730070, Lanzhou, China.

${ }^{2}$ Corresponding author. E-mail: whx1249864076@163.com

Received 15 June 2017; accepted 6 July 2017

Abstract. This paper mainly investigates the oscillation property of the second-order mixed partial difference equation

$$
p u_{m+2, n}+u_{m, n+2}+u_{m, n}+q u_{m+\sigma, n-\tau}=0,
$$

where $\sigma, \tau$ are integers and $\sigma>0, \tau>0$, with $\sigma-\tau-2 \geq 0, m, n$ are non-negative integers, $p$ and $q$ are nonzero constants. And in this paper we describe its oscillatory behavior via envelope theory.

Keywords: Mixed Partial difference equation; 0scillation; Characteristic equation

AMS Mathematics Subject Classification (2010): 39A13

\section{Introduction}

Partial difference equations arise from the study of molecular orbits, mathematical physics problems and the numerical difference approximation problems [1-3]. The investigation of the asymptotic behaviors and oscillations of partial difference equations has attracted more and more attention, see [4-7]. In particular, in [8], Yuan and Liu applying the envelope theory have studied the behavior of oscillation for the following partial difference equation

$$
u_{n+2, m}+u_{n, m+2}+a u_{n+1, m}+b u_{n, m+1}+c u_{n, m}=0
$$

where $a, b, c$ are real numbers and $m, n$ are nonnegative integers. Later in [9], Yuan and Liu investigated the behavior of oscillation for the first-order mixed 2D discret convention system

$$
p u_{m+1, n}+q u_{m, n+1}-u_{m, n}+r u_{m-\sigma, n-\tau}=0,
$$

where $p, q, r$ are real numbers with $p^{2}+q^{2}+r^{2} \neq 0, m, n$ are non-negative integers and $\sigma, \tau$ are nonzero integers with $\sigma \tau<0$.

However, up to now, few paper investigated the oscillation of the second-order mixed partial difference equations. Therefore, in this paper, we will apply envelope theory to investigate the oscillation property for the following partial difference equation 
Hui-li Ma and Hai-xia Wang

$$
p u_{m+2, n}+u_{m, n+2}+u_{m, n}+q u_{m+\sigma, n-\tau}=0
$$

where $\sigma, \tau$ are real numbers and $\sigma>0, \tau>0$, with $\sigma-\tau-2 \geq 0, m, n$ are nonnegative integers, $p$ and $q$ are nonzero constants.

\section{Preliminaries}

In this section, we give some definitions and lemmas which will be used later.

Definition 1. A solution of (1) is a real double sequence $\left\{u_{m, n}\right\}$ which is defined for $m \geq 0, n \geq 0$ and satisfies (1).

Definition 2. A solution $\left\{u_{m, n}\right\}$ of (1) is said to be eventually positive (or negative) if $u_{m, n}>0$ ( (or $u_{m, n}<0$ ) for large numbers $m$ and $n$. It is said to be oscillatory if it is neither eventually positive nor eventually negative. (1) is called oscillatory if all of its nontrivial solutions are oscillatory. We need the following lemma to discuss the oscillation property of (1).

Lemma 1. [10] The following statements are equivalent

(i) Every solution of equation (1) is oscillatory;

(ii) The characteristic equation of (1)

$$
p \lambda^{2}+\mu^{2}+1+q \lambda^{\sigma} \mu^{-\tau}=0
$$

has no positive roots. The following lemma is a powerful tool in this paper.

Lemma 2. [11] Suppose that $f(x, y), g(x, y)$ and $v(x, y)$ are differentiable on $(-\infty,+\infty) \times(-\infty,+\infty)$. Let $\Gamma$ be a two-parameter family of lines defined by the equation

$$
f(\lambda, \mu) x+g(\lambda, \mu) y=v(\lambda, \mu)
$$

where $\lambda$ and $\mu$ are parameters. Let $\Sigma$ be the envelope of the family $\Gamma$. Then the equation

$$
f(\lambda, \mu) a+g(\lambda, \mu) b=v(\lambda, \mu)
$$

has no real roots if and only if there is no tangent line of $\Sigma$ passing through the point $(a, b)$ in $x y$-plane.

\section{Main results}

In this section, some necessary and sufficient conditions for the oscillation properties of all solutions of (1) are established.

Theorem 1. Let $\sigma \geq 1, \tau \geq 1, \sigma-\tau-2=0$. Every solution of (1) oscillates if and only if $p<0, q>0$, and

Proof: The characteristic equation of (1) is

$$
q\left[\frac{-2 p}{(\tau+2) q}\right]^{\frac{\tau+2}{\tau}}+p\left[\frac{-2 p}{(\tau+2) q}\right]^{\frac{2}{\tau}}+1>0
$$


Research on Oscillation Properties for a Class of the Second-order Mixed Partial Difference Equations

$$
p \lambda^{2}+\mu^{2}+1+q \lambda^{\sigma} \mu^{-\tau}=0
$$

for $\sigma=\tau+2$,

$$
p \lambda^{2}+\mu^{2}+1+q \lambda^{\tau+2} \mu^{-\tau}=0
$$

let $\lambda=C \mu, C>0$,

$$
\mu^{2}\left(C^{\tau+2} q+C^{2} p+1\right)+1=0,
$$

(2) has no positive roots if and only if (3) has no positive roots, that is to say,

$$
C^{\tau+2} q+C^{2} p+1 \geq 0
$$

is true forever. Set

$$
f(C)=C^{\tau+2} q+C^{2} p+1 .
$$

Let

$$
f_{C}^{\prime}(C)=0
$$

we can get

$$
C_{0}^{\tau}=\frac{-2 p}{(\tau+2) q}
$$

substitute $C_{0}^{\tau}=\frac{-2 p}{(\tau+2) q}$ into $f_{C}^{\prime \prime}(C)$, and combine with $p<0$, we get

$$
f_{C}^{\prime \prime}\left(C_{0}\right)=-2 p(\tau+1)+2 p=-2 p \tau>0 .
$$

Therefore, $f(C)$ has the unique minimum value

according to Lemma 1, the proof is completed.

$$
f\left(C_{0}\right)=q\left[\frac{-2 p}{(\tau+2) q}\right]^{\frac{\tau+2}{\tau}}+p\left[\frac{-2 p}{(\tau+2) q}\right]^{\frac{2}{\tau}}+1>0,
$$

Theorem 2. Let $\sigma \geq 1, \tau \geq 1, \sigma-\tau-2>0$. Every solution of (1) oscillates if and only if

(i) $p \geq 0, q \geq 0$, or

(ii) $p<0, q>\frac{2(\sigma-\tau-2)^{\frac{(\sigma-\tau-2)}{2}}(-p)^{\frac{\sigma}{2}} \tau^{\frac{\tau}{2}}}{\sigma^{\frac{\sigma}{2}}}$.

Proof: The characteristic equation of (1) is

$$
p \lambda^{2}+\mu^{2}+1+q \lambda^{\sigma} \mu^{-\tau}=0
$$

set

$$
f(p, q, \lambda, \mu)=p \lambda^{2}+\mu^{2}+1+q \lambda^{\sigma} \mu^{-\tau}=0,
$$

according to the envelop theory, the points of the envelope of the two-parameter family of planes defined by (4) satisfy the following equations

$$
\begin{aligned}
& f(x, y, \lambda, \mu)=p \lambda^{2}+\mu^{2}+1+q \lambda^{\sigma} \mu^{-\tau}=0, \\
& f_{\lambda}(x, y, \lambda, \mu)=2 p \lambda+q \sigma \lambda^{\sigma-1} \mu^{-\tau}=0
\end{aligned}
$$


Hui-li Ma and Hai-xia Wang

$$
f_{\mu}(x, y, \lambda, \mu)=2 \mu-q \tau \mu^{-\tau-1} \lambda^{\sigma}=0,
$$

where $\lambda>0$ and $\mu>0$. Eliminating the two parameters $\lambda$ and $\mu$ from (5), we get $x<0$ and the equation of the envelope

$$
\begin{aligned}
& y=\frac{2(\sigma-\tau-2)^{\frac{(\sigma-\tau-2)}{2}}(-x)^{\frac{\sigma}{2}} \tau^{\frac{\tau}{2}}}{\sigma^{\frac{\sigma}{2}}}, \\
& \frac{\partial y}{\partial x}=-\frac{(\sigma-\tau-2)^{\frac{(\sigma-\tau-2)}{2}}(-x)^{\frac{\sigma-2}{2}} \tau^{\frac{\tau}{2}}}{\sigma^{\frac{\sigma-2}{2}}}, \\
& \frac{\partial^{2} y}{\partial x^{2}}=\frac{(\sigma-2)(\sigma-\tau-2)^{\frac{(\sigma-\tau-2)}{2}}(-x)^{\frac{\sigma-4}{2}} \tau^{\frac{\tau}{2}}}{2 \sigma^{\frac{\sigma-2}{2}} .}
\end{aligned}
$$

Since $x<0, y>0, \frac{\partial y}{\partial x}<0, \frac{\partial^{2} y}{\partial x^{2}}>0$, further more $\lim _{x \rightarrow-\infty} y=+\infty$, $\lim _{x \rightarrow 0^{-}} y=0$. Hence, $y$ is a positive and strictly concave function on $(+\infty, 0)$. Thus we can easily see that (1) is oscillating, when the point $(p, q)$ is vertically above the envelope $S$ or in the first quadrant, namely, $p<0, q>\frac{2(\sigma-\tau-2)^{\frac{(\sigma-\tau-2)}{2}}(-p)^{\frac{\sigma}{2}} \tau^{\frac{\tau}{2}}}{\sigma^{\frac{\sigma}{2}}}$, or $p \geq 0, q \geq 0$. Lemma 2 implies the statement of this theorem.

\section{Illustrative examples}

In this section, we give some examples to illustrate the results obtained in Section 3.

Example 1. Consider the partial difference equation

$$
-u_{m+2, n}+u_{m, n+2}+u_{m, n}+u_{m+3, n-1}=0
$$

from (6), we have $p=-1, q=1$ and $\sigma=3, \tau=1$, such that $q\left[\frac{-2 p}{(\tau+2) q}\right]^{\frac{\tau+2}{\tau}}+p\left[\frac{-2 p}{(\tau+2) q}\right]^{\frac{2}{\tau}}+1>0$ and $\sigma-\tau-2=0$, according to Theorem 1 , every solution of (6) is oscillating. The oscillatory behavior of (6) is demonstrated by Figure 1.

Example 2. Consider the partial difference equation

$$
-u_{m+2, n}+u_{m, n+2}+u_{m, n}+u_{m+4, n-1}=0
$$

from (7), we have $p=-1, q=1$ and $\sigma=4, \tau=1$, such that $q>\frac{2(\sigma-\tau-2){ }^{\frac{(\sigma-\tau-2)}{2}}(-p)^{\frac{\sigma}{2}} \tau^{\frac{\tau}{2}}}{\sigma^{\frac{\sigma}{2}}}$ and $\sigma-\tau-2>0$, according to Theorem 2, every 
Research on Oscillation Properties for a Class of the Second-order Mixed Partial Difference Equations

solution of (7) is oscillating. The oscillatory behavior of (7) is demonstrated by Figure 2 .

Example 3. Consider the partial difference equation

$$
2 u_{m+2, n}+u_{m, n+2}+u_{m, n}+2 u_{m+5, n-1}=0
$$

from (8), we have $p=2, q=2$ and $\sigma=5, \tau=1$, such that $p \geq 0, q \geq 0$ and $\sigma-\tau-2>0$, according to Theorem 2 , every solution of (8) is oscillating. The oscillatory behavior of (8) is demonstrated by Figure 3 .

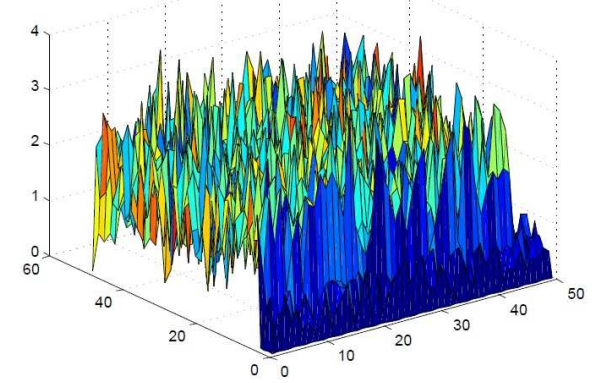

Figure 1: $p=-1, q=1, \sigma=3, \tau=1$

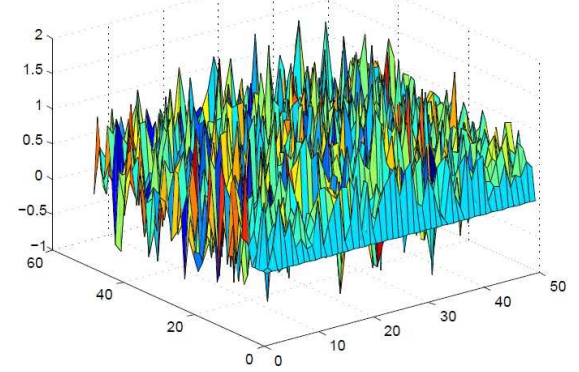

Figure 2: $p=-1, q=1, \sigma=4, \tau=1$

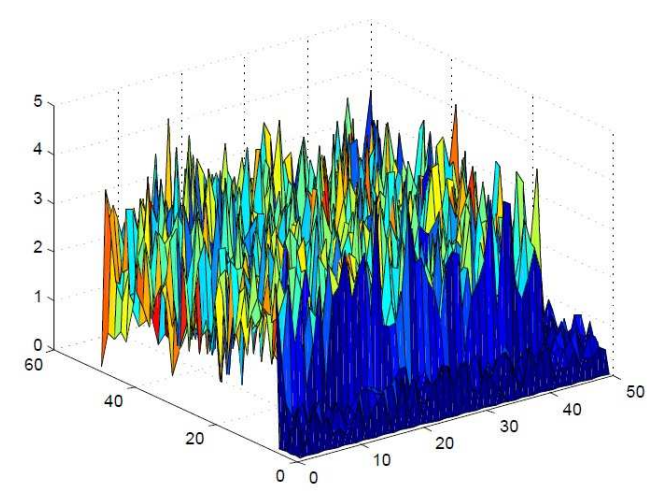

Figure 3: $p=2, q=2, \sigma=5, \tau=1$

\section{REFERENCES}

1. B.G.Zhang and B.M.Liu, Oscillation criteria of certain nonlinear partial difference equations, Comput. Math. Appl., 38 (1999) 107-112.

2. B.G.Zhang and B.M.Liu, Necessary and sufficient conditions for oscillations of partial difference equations with continuous variables, Comput. Math. Appl., 38 (1999) 163-167.

3. R.P.Agarwal, Difference Equations and Inequalities, Marlel Dekker, New York, 1992.

4. R.P.Agarwal and J.Popenda, On the oscillation of recurrence equations, Nonlinear Analysis, 36 (1999) 231-268.

5. W.P.Kelly and A.C.Peterson, Difference Equations, Academic Press, New York, 1991.

6. B.Zhang and R.P.Agarwal, The oscillation and stability of delay partial difference 
Hui-li Ma and Hai-xia Wang

equations, Comput. Math. Appl., 45 (2003) 1253-1295.

7. V.L.J.Kocic and G.Ladas, Global Behavior of Nonlinear Difference Equations of High order with Applications, Klnwer Academic Publishers, Dordrecht, 1993.

8. C.H.Yuan and S.T.Liu, An envelope surface method for determining oscillation of a delay 2-D discrete convection system, J. Differ. Equ., 2014.

9. C.Yuan and S.Liu, Exact regions of oscillation for a mixed 2D discrete convection system, J. Differ. Equ., 2015.

10. B.Zhang and Y.Zhou, Qualitative Analysis of Delay Partial Difference Equations, Hindawi Publishing Corporation, New York, 2007.

11. S.Cheng and Y.Lin, Dual Sets of Envelopes and Characteristic Regions of Quasi-Polynomials, World Scientific, Singapore, 2009.

12. R.P.Agarwal, S.R.Grace and D.Oregan, Oscillation Theory for Difference and Functional Differential Equations, Kluwer Academic, Dordrecht, 2000. 